\title{
Using image analysis techniques to analyze figure- copying performance of patients with visuospatial neglect and control groups
}

\author{
RICHARD GUEST \\ University of Kent, Canterbury, England \\ NICK DONNELLY \\ University of Southampton, Southampton, England \\ MICHAEL FAIRHURST \\ University of Kent, Canterbury, England \\ and \\ JONATHAN POTTER \\ Kent and Canterbury Hospital, Canterbury, England
}

\begin{abstract}
Figure copying is often used to detect visuospatial neglect (VSN) in brain-damaged patients. We describe algorithms that enable the computation of parameters for describing figure-copying performance. The researcher can readily implement these algorithms on a computer using image analysis software, and they provide information on goodness-of-fit, relative to a standard model, as well as on dynamic aspects of subjects' performance in completing figure copies. To demonstrate the clinical utility of these algorithms, preliminary results from a group of right-hemisphere brain-damaged patients, some of whom have VSN, are compared with those for age-matched controls.
\end{abstract}

Often, though not always, patients with visuospatial neglect (VSN) can be discriminated from brain-damaged patients without VSN by their performance on a cancellation task. In such a task, multiple items (usually Xs or lines) are presented on a page, and patients are asked to strike a line through each item. Patients with VSN typically fail to strike lines through (to cancel) many items printed on the left side of the page, whereas they are accurate at striking lines through items printed on the right. Recently, Donnelly et al. (1999) demonstrated that even when the performance of patients with VSN was at ceiling (i.e., all items were canceled), strategic aspects of performance still allowed patients with VSN to be discriminated from both braindamaged control groups without VSN and age-matched control groups. For example, the route taken by subjects in canceling items and the starting points for their cancellations reliably discriminated the subjects with VSN from both control groups. As part of this study, Donnelly et al. presented two cancellation tasks on a graphics tablet attached to a computer, thus demonstrating how algorithms could be written and implemented within the computer software so that the data for studying such strategic factors could be extracted automatically. They concluded that image analysis software, which allows the automatic ex-

Correspondence concerning this article should be addressed to N. Donnelly, Department of Psychology, University of Southampton, University Road, Highfield, Southampton SO17 1BJ, England (e-mail: n.donnelly@soton.ac.uk). traction and categorization of multiple performance parameters, provides an additional tool for detecting VSN. In doing so, they revealed the limitations of traditional paper-and-pencil versions of standard clinical tests.

The present paper describes an extension of this research program. Like cancellation tasks, figure copying is frequently used as a task to detect the presence of VSN (see Bradshaw \& Mattingley, 1995). Copies drawn by patients with VSN can, on occasion, show omissions of significant parts of figures, although such omissions are not always made. Of course, the case of figure copying is more complex than that of cancellation; problems in figure copying can also result from constructional apraxia, as measured by drawing dysfunction. Research has shown that drawing dysfunction follows both posterior and anterior brain damage (e.g., Marshall et al., 1994), with damage to the parietal cortex being associated with both VSN and drawing dysfunction. Marshall et al. proposed that in the case of posterior (parietal) lesions, a common visuospatial mechanism is disturbed that can, depending on severity, lead to problems just in the production of recognizable drawings or VSN. To the extent that this is true, evidence of VSN might be found in the copying process, even when copies are complete.

It is also the case that figure copying is a complex task that requires coordinated actions and multiple attentional fixations. Researchers have investigated the link between disorders of spatial attention (thought to be a key factor whose damage leads to VSN) and action. Patients with 
RH brain damage were slower in making leftward movements than were controls (Mattingley, Phillips, \& Bradshaw, 1994), had to correct skewed movement trajectories to achieve success in pointing and bisection tasks (Goodale, Milner, Jakobson, \& Carey, 1990), and had eye-movements that restricted analysis to the right side of figures (Karnath, 1994). In other words, copying is a complex task that requires control of eye, arm, and hand movements, all of which have been shown to be affected by RH brain damage and to present, specifically, in patients with VSN. It is reasonable to suppose that these factors affecting the production of actions should be manifest during figure copying and should affect subjects' performance in a measurable way.

The questions addressed here are whether (1) algorithms can be written to capture important aspects of the process and completion (or partial completion) of figure copying, (2) figure copying can be implemented on a graphics tablet (attached to a computer) allowing analysis of real figure copies, and (3) the output of these algorithms will allow patients with VSN to be differentiated from control groups even when their figure copies are complete. Given the recent evidence of VSN as a multifaceted disorder, a major objective of this work is to transform standard clinical tests so that they can automatically reveal data thought to be relevant from evidence-based research. Traditional paper-and-pencil tests are limited in their outcome measures and their utility. By implementing paper-and-pencil tests so that image analysis software automatically codes important parameters, the utility of basic clinical tests should be enhanced.

\section{Experimental Methodology}

Four geometric figure-copying tasks were given to subjects. Two of these shapes (the cross and the cube) had been used in a copying study by Warrington, James, and Kinsbourne (1966); the two other shapes were selected for their simplicity, signified by the low number of sides (the square), and their relative complexity (the five pointed star). Three of the shapes were representations of 2-D figures (square, cross, and star), and the fourth was a representation of a 3-D figure (cube). The shapes were presented individually toward the top and in the center of a landscape-oriented A4 sheet. Image sizes (maximum width $\times$ maximum height) were $21 \times 21 \mathrm{~mm}$ for the square, $42 \times 42 \mathrm{~mm}$ for the cross, $22 \times 22 \mathrm{~mm}$ for the star, and $36 \times 36 \mathrm{~mm}$ for the cube. As in standard figurecopying tasks, the subjects were required to copy each shape. The copies were made on the piece of paper with the printed figure. The subjects were instructed to complete their copies below the printed shape. The order in which the shapes were presented (square, cross, star, and cube) remained constant for all subjects.

\section{Algorithms for Capturing Performance on Figure-Copying Tasks}

Drawing performance was analyzed in two basic ways. First, the copy completed by the participant was judged against the geometric and topological properties of the target image. This analysis of the static properties of figure copies was conducted using both a categorical pass/fail criterion and qualitative criteria based on the accuracy of various measured parameters.

Second, performance was measured during the production of figure copies. We refer to this as analyzing dynamic aspects of performance. Below we outline algorithms for the analysis of both static and dynamic performance. The algorithms for measuring static performance are shape specific and are described separately for each shape.

\section{Static Features}

Shapes. Rule-based assessment criteria were used to standardize the assessment of the drawn shapes, using a component present elements methodology (Wilson, Cockburn, \& Halligan, 1987). The following rules defined each shape on the basis of the number of identifiable components within each shape and the location of reference points. Drawings not complying with the component presence/layout rules defined below were deemed to have "failed." Figure 1 shows the constituent parts for and reference locations for each of the shapes.

Square. Copies were complete if they contained four clearly defined individual edge components. Edges did not need to form corners, and adjacent sides did not need to be perpendicular, because these qualitative features were assessed in terms of static features measuring corner formation and relative spatial positioning of corner locations using the corner points within the copying model for comparison. Number of components: four edges.

Cross. Copies were complete if the five sub-boxes that formed the cross were present. As with the square, the sides of each box did not need to be perpendicular. However, the boxes had to form a cross shape. Each box had to comprise four sides. The four internal corners of the cross were used as spatial model comparison points. Number of components: five sub-boxes.

Star. Copies were complete if the drawn shape contained five acute external vertices and an internal pentagon. The five external vertices were used as spatial model comparison points. Number of components: five external vertices.

Cube. Copies were complete if the drawing contained seven vertices, four forming the "square" section of the cube (the square formation rules defined above are applied) and three others forming the top left, top right, and bottom right of the 3-D section of the drawing. Number of components: seven internal vertices.

Figure 2 shows some examples of responses passing and failing the rule-based assessment criteria for the square-copying task. Figure 2A shows drawings satisfying the rule-based inclusion criteria for the square; the nonperpendicularity of the sides and the inaccurate joining at corners did not fail the shape. Figure 2B shows responses that failed the inclusion criteria by either omitting a component or perseveration (multiple drawing of a single component) within an edge. 

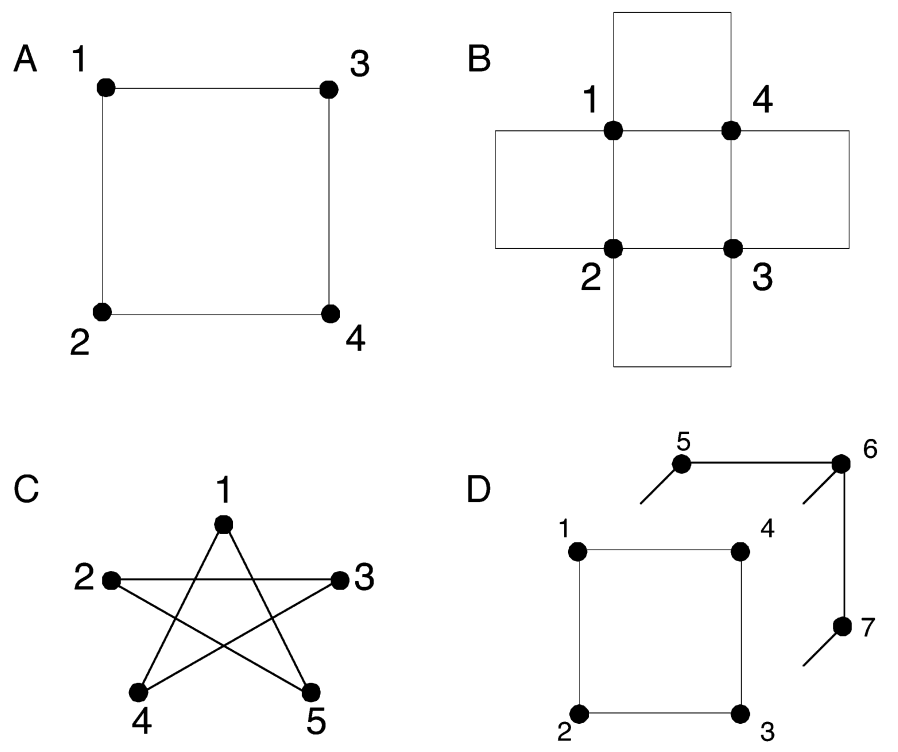

Figure 1. Reference points within shape models.

In addition to the pass/fail criteria resulting from the analysis of basic components, other algorithms were used to measure a range of different static features that, taken together, quantify the quality of figure copies.

Drawing segments. This measure of the number of times the pen was removed from the tablet during the drawing time (not including the final pen lift at the end of the drawing; hence the deduction of a single occurrence within the calculation) gives the number of movement segments within the drawing.

$$
\text { Segments }=\left(\sum_{t=0}^{n}\{[Z(t-1) \rightarrow Z(t)]=(1 \rightarrow 0)\}\right)-1,
$$

where $n=$ number of packets, $Z(t-1)=$ pen tip status at time $t-1, Z(t)=$ pen tip status at time $t, Z(t)=0=$ pen tip not on tablet, and $Z(t)=1=$ pen tip on tablet.
Starting point (SP). Drawing responses were divided into left, center, and right regions. Dividing the spatial extent of each copy by three gave the left, center, and right sections to each copy. SP was relative to the finished copy (whether compete or incomplete) rather than relative to specific features copied.

It is also possible to measure other parameters using this method, and these have been implemented in our software. We describe these parameters separately, because the data generated are not referred to in the case study described below.

Mean reference positional error (mm). Using the reference points within the individual shape models, the mean reference point positional error was calculated from the Euclidean distance between pairs of adjacent corner reference points located within the drawings. Corners within the drawn image were defined at inter-

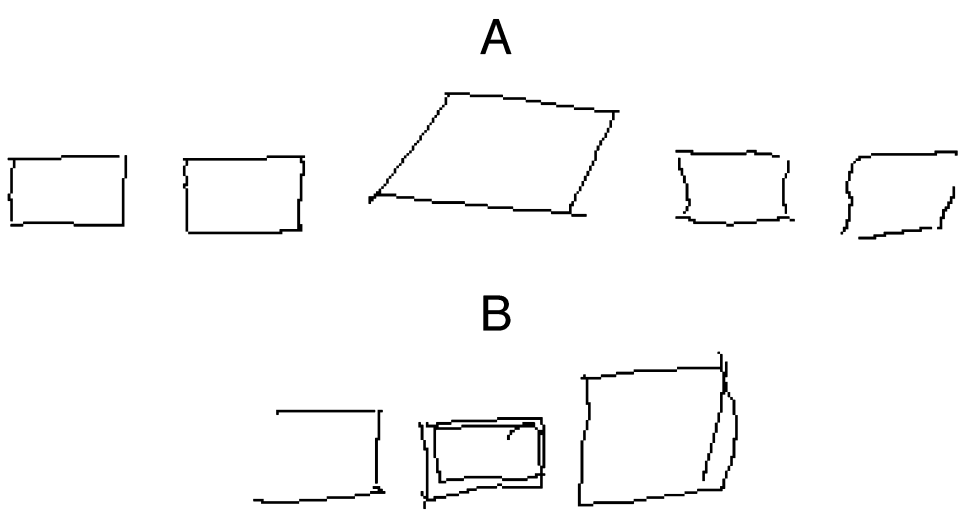

Figure 2. Examples of figure copies passing (A) and failing (B) the rule-based assessment criteria. 
sections or at an interpolation of the intersection point if lines did not cross. A mean was taken of the differences between the model and the set of calculated distances to produce a result (in millimeters) describing the drawing accuracy.

Width and height model differences $(\mathbf{m m})$. The width and height of the drawing response was calculated from the maximum and minimum drawn coordinates on the $y$ - and $x$-axes, respectively. Deviations from the model width and height provided a copying accuracy measure.

Total distance ( $\mathbf{m m})$. The total distance, the pen travel distance while on the tablet, was calculated by summing the Euclidian distance between pairs of coordinates.

\section{Dynamic Features}

Two main strands of dynamic feature were extracted from the test responses detailing timing and pen kinematic aspects of the drawing performance. The features are defined below:

Execution time (sec). This was the time period when the pen was drawing on the surface of the tablet calculated by

$$
\text { Execution Time }=t_{\text {end }}-t_{\text {start }},
$$

where $t_{\text {end }}=$ final time at which the pen tip was on the graphics tablet surface for individual shape and $t_{\text {start }}=$ time of initial pen tip contact.

Mean velocity $(\mathbf{m m} / \mathbf{s e c})$. Pen velocity across the surface of the tablet was calculated by taking the first derivative of the coordinate pair displacement against time. Third order, four coefficient polynomial modeling was used to obtain a derivative of displacement at each coordinate point (Williams, 1986). Using a constant sampling time-base, the following approximation used displacement values of four sets of coordinates at times $t-2, t-1, t+1$, and $t+2$ :

$$
\frac{d s}{d t} \approx \frac{1}{12}\left(-s_{t+2}+8 s_{t+1}-8 s_{t-1}+s_{t-2}\right) .
$$

The displacements used within the calculation were extracted by calculating the Euclidean displacement from both the $x$ and $y$ components. The mean velocity was obtained by summing the velocities at individual points within the drawing and dividing by the number of samples taken.

$$
\text { Mean velocity }=\frac{\sum_{t=0}^{n}\left(\frac{d s}{d t}\right)}{n},
$$

where $n=$ the number of samples. Velocities were calculated only when the pen was on the tablet surface.

Mean acceleration $\left(\mathbf{m m} / \mathbf{s e c}^{2}\right)$. Pen acceleration is the second derivative of the coordinate displacement. The third order, four coefficient polynomial approximation for acceleration was given by

$$
\frac{d^{2} s}{d t^{2}} \approx \frac{1}{3}\left(s_{t+2}-s_{t+1}-s_{t-1}+s_{t-2}\right)
$$

As with the mean velocity calculation, mean acceleration was calculated by summing the acceleration at individual points within the drawing and dividing by the number of samples taken.

Pen contact ratio. This ratio indicates the proportion of the overall task execution time when the pen is actually drawing on the tablet surface. It is calculated with the following formula:

$$
\text { Pen contact ratio }=\frac{\text { Total time pen off tablet }}{\text { Total time pen on tablet }} \text {. }
$$

\section{Data Capture}

With the exception of mean velocity and acceleration, all of the algorithms detailed above could be measured manually, although this would be a laborious process. However, in our implementation of figure-copying tasks, subjects completed their copies with the paper sheets fixed and resting on a Wacom WD1212 graphics digitization tablet (spatial accuracy of 180 points/cm in $x$ - and $y$-axes) connected to a portable computer. As the pen moved across the tablet surface during figure copying, the coordinates of the pen were transmitted to the computer at a baud rate of $19200 \mathrm{bits} / \mathrm{sec}$. These data (along with other data regarding pen tilt and pressure) were time stamped by the computer at a rate of $100 \mathrm{~Hz}$ and stored in an ASCII response file. Capturing the data in this fashion enabled the algorithms to be implemented within a computer program and for all analyses to be conducted automatically.

Note that the analysis was not performed in real time but on stored data files. The main benefits of storing data in this form (as opposed to real-time analysis) are twofold. First, complex feature extraction routines can be used on the data without the need to buffer the real-time data stream from the graphics tablet. Second, responses can be reanalyzed so that new features can be defined and extracted without the need for retesting.

\section{Preliminary Case Study}

The important question that stems from this work is whether automated algorithms work to indicate VSN when faced with real images drawn by participants with a wide range of drawing competencies, not least because some participants have VSN and others may have constructional problems resulting from brain damage. If the parameters generated by these algorithms allowed further differentiation of patients with VSN from controls, relative to that allowed by visual inspection alone, then image analysis techniques should be considered in the design of clinical assessment tools.

Our initial test compared two groups of right-hemisphere brain-damaged patients (with and without VSN) and a group of age-matched controls. The patient group received CT scans, and data on the localization of brain damage are presented in Table 1. Patients with VSN were identified using the Conventional battery of the Behavioural Inattention Test (BIT; Wilson et al., 1987); 11 were male, 9 female, with a mean age of $76.94(S D=$ 
7.10; range, 90-64). The mean BIT score obtained by the VSN group was 89.8 (range, 41-128). Brain-damaged patients without VSN (i.e., scoring above 129 in the Conventional BIT battery) were included in the stroke control test group (SC). Thirty-nine subjects (21 male, 18 female) were included in this group, with a mean age of $74.62(S D=7.87$; range, 92-60) and a mean BIT score of 143.56. In addition, a series of 13 healthy age-matched control subjects (AMC) with no known history of vascular disease were tested; 5 were male and 8 female, with a mean age of $72.77(S D=4.07$; range, $79-63)$. No BIT assessment was administered to the AMC group. In comparing these groups, the question was whether the pass/fail rule-based assessment criteria could differentiate $\mathrm{SC}$ and AMC control groups from patients with VSN. The pass/fail rule-based assessment criteria showed that the patients with VSN and the SC group consistently failed to draw copies as accurately as the AMC group (see Figure 3). However, they also showed that the star was failed by some in the AMC group. In fact, the differences in frequencies between groups passing or failing to copy the star were not significant $\left[\chi^{2}(2)=3.2\right]$. In contrast, the square, cross, and cube were copied correctly by most SCs and all AMCs. The differences in frequencies between groups passing or failing to copy the square and cross were significant $\left[\chi^{2}(2)=6.6,15.76\right.$, and $8.35, p<.001$ for the square, cross, and cube, respectively]. Importantly, these significant results held when the AMC group was removed from the analyses and the comparison was made between the VSN and SC groups $\left[\chi^{2}(1)=3.3,8.4\right.$, and $4.5, p<.05$ for the square and cube, and $p<.01$ for the cross]. Together, these data indicate that the square, cube, and cross discriminated between patients with and without VSN and AMC, although the copying of the cross provided the better discrimination. In addition, reanalyzing these data with re-

Table 1

Patient and Age-Matched Control Data

\begin{tabular}{|c|c|c|c|c|c|c|c|c|c|c|c|c|c|c|c|}
\hline Gender & Age & BIT & Damage & Square & Cross & Cube & Star Start & Gender & Age & BIT & Damage & Square & Cross & Cube & Star Start \\
\hline \multicolumn{8}{|c|}{ Brain-Damaged Patients With Posterior Damage } & \multicolumn{8}{|c|}{ Brain-Damaged Patients Without Posterior Damage } \\
\hline Female & 90 & 41 & $\mathrm{~T}-\mathrm{O}-\mathrm{S}$ & $\mathrm{P}$ & $\mathrm{F}$ & $\mathrm{F}$ & $\mathrm{L}$ & Female & 85 & 79 & $\mathrm{~S}$ & $\mathrm{~F}$ & $\mathrm{~F}$ & $\mathrm{P}$ & $\mathrm{C}$ \\
\hline Male & 69 & 53 & $\mathrm{~T}-\mathrm{P}-\mathrm{O}$ & $\mathrm{P}$ & $\mathrm{F}$ & $\mathrm{F}$ & $\mathrm{L}$ & Female & 85 & 80 & $\mathrm{~S}$ & $\mathrm{~F}$ & $\mathrm{~F}$ & $\mathrm{P}$ & $\mathrm{C}$ \\
\hline Male & 75 & 61 & $\mathrm{O}$ & $\mathrm{P}$ & $\mathrm{F}$ & $\mathrm{F}$ & $\mathrm{L}$ & Female & 77 & 110 & $\mathrm{~S}$ & $\mathrm{P}$ & $\mathrm{F}$ & $\mathrm{F}$ & $\mathrm{L}$ \\
\hline Female & 74 & 62 & T-P & $\mathrm{P}$ & $\mathrm{P}$ & $\mathrm{F}$ & $\mathrm{L}$ & Male & 80 & 110 & $\mathrm{~S}$ & $\mathrm{P}$ & $\mathrm{P}$ & $\mathrm{F}$ & $\mathrm{L}$ \\
\hline Male & 72 & 67 & $\mathrm{O}-\mathrm{S}$ & $\mathrm{F}$ & $\mathrm{F}$ & $\mathrm{F}$ & $\mathrm{L}$ & Female & 75 & 122 & $\mathrm{~S}$ & $\mathrm{~F}$ & $\mathrm{~F}$ & $\mathrm{~F}$ & $\mathrm{~L}$ \\
\hline Female & 86 & 67 & T-P & $\mathrm{P}$ & $\mathrm{F}$ & $\mathrm{F}$ & $\mathrm{L}$ & Male & 76 & 128 & $\mathrm{~S}$ & $\mathrm{P}$ & $\mathrm{P}$ & $\mathrm{P}$ & $\mathrm{C}$ \\
\hline Male & 75 & 76 & $\mathrm{P}$ & $\mathrm{P}$ & $\mathrm{F}$ & $\mathrm{F}$ & $\mathrm{L}$ & Male & 72 & 134 & $\mathrm{~S}$ & $\mathrm{~F}$ & $\mathrm{~F}$ & $\mathrm{~F}$ & $\mathrm{~L}$ \\
\hline Male & 71 & 82 & $\mathrm{P}$ & $\mathrm{F}$ & $\mathrm{P}$ & $\mathrm{F}$ & $\mathrm{L}$ & Female & 79 & 135 & $\mathrm{~S}$ & $\mathrm{P}$ & $\mathrm{P}$ & $\mathrm{P}$ & $\mathrm{C}$ \\
\hline Male & 68 & 96 & F-P-O & $\mathrm{P}$ & $\mathrm{F}$ & $\mathrm{F}$ & $\mathrm{L}$ & Female & 72 & 136 & $\mathrm{~S}$ & $\mathrm{P}$ & $\mathrm{P}$ & $\mathrm{F}$ & $\mathrm{L}$ \\
\hline Female & 75 & 101 & $\mathrm{TP}$ & $\mathrm{P}$ & $\mathrm{P}$ & $\mathrm{P}$ & $\mathrm{C}$ & Male & 70 & 140 & $\mathrm{~S}$ & $\mathrm{P}$ & $\mathrm{F}$ & $\mathrm{F}$ & $\mathrm{L}$ \\
\hline Female & 77 & 105 & $\mathrm{P}$ & $\mathrm{P}$ & $\mathrm{P}$ & $\mathrm{F}$ & $\mathrm{L}$ & Male & 75 & 142 & $\mathrm{~S}$ & $\mathrm{P}$ & $\mathrm{F}$ & $\mathrm{F}$ & $\mathrm{L}$ \\
\hline Male & 64 & 109 & $\mathrm{P}$ & $\mathrm{P}$ & $\mathrm{F}$ & $\mathrm{F}$ & $\mathrm{L}$ & Male & 78 & 142 & $\mathrm{~F}$ & $\mathrm{P}$ & $\mathrm{F}$ & $\mathrm{P}$ & $\mathrm{C}$ \\
\hline Male & 88 & 123 & $\mathrm{P}$ & $\mathrm{P}$ & $\mathrm{F}$ & $\mathrm{P}$ & $\mathrm{C}$ & Male & 78 & 143 & NSL & $\mathrm{P}$ & $\mathrm{P}$ & $\mathrm{F}$ & $\mathrm{L}$ \\
\hline Male & 75 & 124 & $\mathrm{P}$ & $\mathrm{P}$ & $\mathrm{F}$ & $\mathrm{F}$ & $\mathrm{L}$ & Male & 84 & 144 & $\mathrm{~S}$ & $\mathrm{P}$ & $\mathrm{P}$ & $\mathrm{P}$ & $\mathrm{C}$ \\
\hline Female & 78 & 132 & $\mathrm{P}-\mathrm{O}$ & $\mathrm{P}$ & $\mathrm{F}$ & $\mathrm{F}$ & $\mathrm{L}$ & Male & 83 & 145 & NSL & $\mathrm{F}$ & $\mathrm{F}$ & $\mathrm{F}$ & $\mathrm{L}$ \\
\hline Female & 82 & 133 & $\mathrm{P}$ & $\mathrm{P}$ & $\mathrm{P}$ & $\mathrm{P}$ & $\mathrm{C}$ & Female & 67 & 146 & $\mathrm{~S}$ & $\mathrm{P}$ & $\mathrm{F}$ & $\mathrm{F}$ & $\mathrm{L}$ \\
\hline Male & 84 & 133 & T-P-O & $\mathrm{P}$ & $\mathrm{P}$ & $\mathrm{P}$ & $\mathrm{C}$ & Female & 72 & 146 & $\mathrm{~S}$ & $\mathrm{P}$ & $\mathrm{F}$ & $\mathrm{P}$ & $\mathrm{C}$ \\
\hline Male & 73 & 135 & $\mathrm{P}$ & $\mathrm{P}$ & $\mathrm{F}$ & $\mathrm{F}$ & $\mathrm{L}$ & Female & 63 & 146 & $\mathrm{~S}$ & $\mathrm{P}$ & $\mathrm{F}$ & $\mathrm{F}$ & $\mathrm{L}$ \\
\hline Male & 77 & 138 & R-T-P & $\mathrm{P}$ & $\mathrm{F}$ & $\mathrm{F}$ & $\mathrm{L}$ & Male & 74 & 146 & $\mathrm{~S}$ & $\mathrm{P}$ & $\mathrm{P}$ & $\mathrm{P}$ & $\mathrm{C}$ \\
\hline Male & 61 & 139 & T-P-O & $\mathrm{P}$ & $\mathrm{F}$ & $\mathrm{F}$ & $\mathrm{L}$ & Male & 77 & 146 & $\mathrm{~S}$ & $\mathrm{P}$ & $\mathrm{P}$ & $\mathrm{F}$ & $\mathrm{L}$ \\
\hline Female & 69 & 139 & T-P-O & $\mathrm{P}$ & $\mathrm{P}$ & $\mathrm{P}$ & $\mathrm{C}$ & Male & 71 & 146 & $\mathrm{~S}$ & $\mathrm{~F}$ & $\mathrm{P}$ & $\mathrm{F}$ & $\mathrm{L}$ \\
\hline Male & 60 & 143 & $\mathrm{~T}-\mathrm{P}$ & $\mathrm{P}$ & $\mathrm{F}$ & $\mathrm{P}$ & $\mathrm{C}$ & Female & 68 & 146 & $\mathrm{~S}$ & $\mathrm{P}$ & $\mathrm{P}$ & $\mathrm{P}$ & $\mathrm{C}$ \\
\hline Female & 78 & 143 & $\mathrm{P}$ & $\mathrm{P}$ & $\mathrm{P}$ & $\mathrm{P}$ & $\mathrm{C}$ & Female & 77 & 146 & $\mathrm{~F}$ & $\mathrm{~F}$ & $\mathrm{~F}$ & $\mathrm{P}$ & $\mathrm{C}$ \\
\hline Female & 83 & 145 & $\mathrm{~T}$ & $\mathrm{P}$ & $\mathrm{P}$ & $\mathrm{F}$ & $\mathrm{L}$ & Male & 83 & 146 & NSL & $\mathrm{P}$ & $\mathrm{F}$ & $\mathrm{P}$ & $\mathrm{C}$ \\
\hline Female & 82 & 145 & P-S & $\mathrm{P}$ & $\mathrm{P}$ & $\mathrm{P}$ & $\mathrm{C}$ & \multirow{2}{*}{\multicolumn{8}{|c|}{ Age-Matched Controls }} \\
\hline Female & 60 & 146 & $\mathrm{P}$ & $\mathrm{P}$ & $\mathrm{F}$ & $\mathrm{F}$ & $\mathrm{L}$ & & & & & & & & \\
\hline Female & 73 & 146 & T-P-O & $\mathrm{P}$ & $\mathrm{P}$ & $\mathrm{P}$ & $\mathrm{C}$ & Female & 69 & - & - & $\mathrm{P}$ & $\mathrm{P}$ & $\mathrm{P}$ & $\mathrm{L}$ \\
\hline Male & 61 & 146 & T-P-O-S & $\mathrm{P}$ & $\mathrm{F}$ & $\mathrm{P}$ & $\mathrm{C}$ & Female & 63 & - & - & $\mathrm{P}$ & $\mathrm{P}$ & $\mathrm{P}$ & $\mathrm{L}$ \\
\hline Male & 77 & 146 & $\begin{array}{l}P \\
1-1-0-3\end{array}$ & $\mathrm{P}$ & $\mathrm{F}$ & $\mathrm{P}$ & $\mathrm{C}$ & Female & 76 & - & - & $\mathrm{P}$ & $\mathrm{P}$ & $\mathrm{P}$ & $\mathrm{C}$ \\
\hline Female & 83 & 146 & $\mathrm{P}$ & $\mathrm{P}$ & $\mathrm{P}$ & $\mathrm{P}$ & $\mathrm{C}$ & Female & 72 & - & - & $\mathrm{P}$ & $\mathrm{P}$ & $\mathrm{P}$ & $\mathrm{C}$ \\
\hline Female & 92 & 146 & F-P-S & $\mathrm{P}$ & $\mathrm{F}$ & $P$ & C & Female & 71 & - & - & $\mathrm{P}$ & $\mathrm{P}$ & $\mathrm{P}$ & $\mathrm{L}$ \\
\hline Male & 62 & 146 & 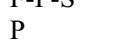 & $\mathrm{P}$ & $\mathrm{F}$ & $P$ & C & Female & 79 & - & - & $\mathrm{P}$ & $\mathrm{P}$ & $\mathrm{P}$ & $\mathrm{C}$ \\
\hline Female & 72 & 146 & $\mathrm{P}$ & $\mathrm{P}$ & $\mathrm{F}$ & $\mathrm{F}$ & L & Female & 77 & - & - & $\mathrm{P}$ & $\mathrm{P}$ & $\mathrm{P}$ & $\mathrm{L}$ \\
\hline Male & 85 & 146 & $\mathrm{P}$ & $\mathrm{P}$ & $\begin{array}{l}\Gamma \\
P\end{array}$ & $\begin{array}{l}\Gamma \\
P\end{array}$ & $\begin{array}{l}\mathrm{L} \\
\mathrm{C}\end{array}$ & Female & 75 & - & - & $\mathrm{P}$ & $\mathrm{P}$ & $\mathrm{P}$ & $\mathrm{C}$ \\
\hline Male & 75 & 146 & O-P & $\mathrm{F}$ & $\mathrm{F}$ & $\mathrm{F}$ & $\mathrm{L}$ & Male & 66 & - & - & $\mathrm{P}$ & $\mathrm{P}$ & $\mathrm{P}$ & $\mathrm{L}$ \\
\hline & 15 & 140 & $U-P$ & $\mathrm{~F}$ & $\mathrm{~F}$ & $\mathrm{~F}$ & L & Male & 75 & - & - & $\mathrm{P}$ & $\mathrm{P}$ & $\mathrm{P}$ & $\mathrm{C}$ \\
\hline & & & & & & & & Male & 73 & - & - & $\mathrm{P}$ & $\mathrm{P}$ & $\mathrm{P}$ & $\mathrm{L}$ \\
\hline & & & & & & & & Male & 76 & - & - & $\mathrm{P}$ & $\mathrm{P}$ & $\mathrm{P}$ & $\mathrm{L}$ \\
\hline & & & & & & & & Male & 74 & - & - & $\mathrm{P}$ & $\mathrm{P}$ & $\mathrm{P}$ & $\mathrm{L}$ \\
\hline
\end{tabular}

Note-For brain damage: $\mathrm{F}=$ frontal; $\mathrm{O}=$ occipital; $\mathrm{P}=$ parietal; $\mathrm{T}=$ temporal; $\mathrm{S}=$ subcortical; NSL = no specific localization. Elsewhere $\mathrm{P}=$ pass; $\mathrm{F}=$ fail; $\mathrm{L}=$ left; and $\mathrm{C}=$ center/right. Pass/fail data are given for square, cross, and cube copies, and left versus center (and right) starting point data are given for star copies. Patients scoring 129 or less on the BIT were considered to have VSN. 


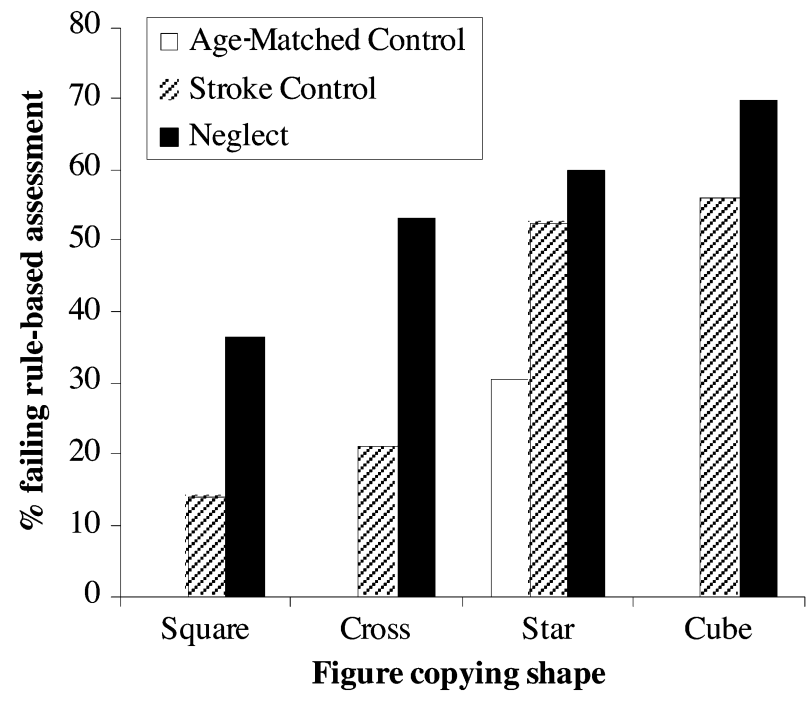

Figure 3. Percentage of each group excluded according to the rule-based assessment criteria.

spect to posterior damage (i.e., damage including that to the temporal, parietal, or occipital lobes) versus frontal and subcortical damage demonstrated that posterior patients failing the BIT were much more likely to fail the cube-copying task than those passing the BIT [0.77 vs. $\left.0.2 ; \chi^{2}(1)=10.45, p<.01\right]$. No such effect of passing or failing the BIT on the success of cube copying was evident in the frontal/subcortical group [0.35 vs. 0.71 ; $\left.\chi^{2}(1)=2.25\right]$. No similar contrasts were significant in square or cross copying ( $\chi^{2}<1$ in both cases). In summary, where posterior brain-damage was involved, failure at cube copying was highly indicative of failing the BIT.

Our previous analysis of performance in cancellation tasks suggests that overall execution time and starting position might have differentiated the VSN and SC groups. In the present context, execution time and related variables of velocity and acceleration are continuous variables, whereas starting point is not. Therefore, to investigate starting point, the VSN and SC groups were compared for the likelihood of starting in the left, center, or right of a copy. To investigate the impact of VSN on other measures, we investigated the correlations between the score on the BIT and other variables.

\section{Starting Position}

Few subjects began copying on the right side of the figures, so these data were summed with those from patients starting in the center to form two levels of the starting point category, starting on the left versus starting on the center and right. The result of these $2 \times 2$ chi-squared analyses demonstrated a highly significant effect of starting point for copies of stars $\left[\chi^{2}(1)=14.70, p<.01\right]$ but no significant effects for copies of squares $\left[\chi^{2}(1)<1\right]$, cubes $\left[\chi^{2}(1)<1\right]$, or crosses $\left[\chi^{2}(1)=2.23\right]$. In the case of copying stars, patients with VSN tended to start on the left and move rightward, whereas the SC group tended to start in the center. Therefore, starting point can differentiate brain-damaged patients with VSN from SC patients when they copy stars but not when they copy the other figures tested.

The effect of starting position was recomputed for the star-copying task (see Table 2) after splitting the VSN and SC groups into posterior groups (i.e., damage including temporal, parietal, or occipital lobes) and groups with exclusively frontal and/or subcortical damage. The analysis revealed that posterior VSN patients were much more likely to start on the left than were posterior SC patients [.71 vs. .20, $\left.\chi^{2}(1)=7.74\right]$. In contrast, no such effect of passing or failing the BIT was evident for the frontal/subcortical group [0.35 vs. .29; $\left.\chi^{2}(1)<1\right]$.

To investigate the impact of VSN on dynamic measures, the BIT scores for all brain-damaged patients were correlated with their scores on each of the dynamic and static measures using Spearman rank-order correlations with Bonferroni adjusted significance levels adjusted (see Table 2). There were significant negative correlations between the BIT and execution time, as well as positive correlations between the BIT and acceleration, for

Table 2

Spearman Rank-Order Correlations Between BIT Score and Dynamic Performance Measures for All Patients, Patients With Posterior Brain Damage, and Patients With Nonposterior Brain Damage

\begin{tabular}{|c|c|c|c|c|c|c|}
\hline Subjects & Shape & DS & PCR & ET & Velocity & Acceleration \\
\hline \multirow[t]{4}{*}{ All } & Square & -.177 & -.148 & -.321 & .322 & .384 \\
\hline & Cross & -.094 & -.24 & -.329 & .256 & .387 \\
\hline & Cube & .385 & .129 & .391 & -.308 & -.442 \\
\hline & Star & -.293 & -.353 & -.201 & -.061 & .11 \\
\hline \multirow[t]{4}{*}{ Posterior } & Square & -.194 & -.081 & -.264 & .341 & .337 \\
\hline & Cross & -.134 & -.298 & -.302 & .286 & .384 \\
\hline & Cube & .471 & .154 & .535 & -.387 & -.608 \\
\hline & Star & -.368 & -.484 & -.232 & -.095 & .129 \\
\hline \multirow[t]{4}{*}{ Nonposterior } & Square & -.293 & -.354 & -.521 & .316 & .535 \\
\hline & Cross & -.055 & -.081 & -.503 & .222 & .431 \\
\hline & Cube & .197 & .041 & .091 & -.313 & -.211 \\
\hline & Star & -.14 & -.05 & -.086 & -.065 & .077 \\
\hline
\end{tabular}

Note-DS, drawing segments; PCR, pen contact ratio; ET, execution time. Significant correlations (after application of Bonferroni correction, $p<.05 / 20$ ) are shown in bold type. 
copying the square and the cross. These data suggest that low scores on the BIT lead to slower copying of squares and cubes, where movement speeds are less variable. Recomputing these correlations after splitting the patient group into a posterior group (i.e., damage including that to temporal, parietal, or occipital lobes) and a group with exclusively frontal and/or subcortical damage showed the patterns of correlations to be true for both groups.

In contrast, significant positive correlations between the BIT and drawing segments (DS) and execution time (ET) and significant negative correlations between the BIT and acceleration suggest that copying the cube represents a different problem. Patients scoring highly on the BIT drew fewer segments, drew at a more constant speed, and took less time than did those scoring highly on the BIT. Importantly, when these correlations were recomputed with the patients separated into those with posterior damage versus those with exclusively frontal and/or subcortical damage, the correlations were significant only for the posterior group, and in the case of ET and mean acceleration (MA), there was a strong trend for significantly bigger correlations in the posterior than in the frontal and/or subcortical group $(z=1.76$ and 1.81 for MA and ET, respectively). These data suggest that posterior lesions influenced cube copying more than did frontal/subcortical lesions, further supporting the pass/fail data for cube copying.

\section{Discussion}

The aims of the research reported here were to investigate whether algorithms could be defined that would enhance the detection of VSN from performance on figurecopying tasks. Furthermore, we wished to investigate whether these algorithms could be made so explicit that they could be written as computer code and employed using image analysis techniques. We have shown that it is possible to define algorithms that can be implemented within image analysis software and that this software can be used to reliably categorize copies as being recognizable representations of target figures. In the cases of squares, crosses, and cubes, the likelihood of successful copies' being recorded is predicted by passing or failing the BIT. Consideration of the starting point for copying demonstrated that VSN patients were likely to begin copying on the left side of stars but not of other figures.

In an attempt to demonstrate that the copying tasks can inform issues of localization, the patient group was divided into posterior and frontal/subcortical groups. The data showed that both cube copying and starting position star copying were sensitive to the location of brain damage. In fact, one can score cube copying and starting position as two binary variables, with passing the cube task and starting copying stars in the center (or right) receiving scores of 1 , while failing the cube task and starting copying stars on the right receive scores of 2 . Adding together these two scores and subjecting them to a oneway analysis of variance with four groups (posterior and frontal/subcortical VSN and SC groups) revealed a main effect of group $[F(1,58)=7.7, p<.01]$; Bonferroni cor- rected comparisons showed that patients with posterior damage failed the BIT score significantly more highly than did all other groups. No other comparisons were significant. In other words, performance on the cubeand star-copying tasks indicated a significant likelihood of posterior brain damage if the cube-copying task was failed and the star-copying task was begun on the left side.

The algorithms were also able to detect dynamic features influenced by VSN as measured by the BIT. For copying squares and crosses, this influence was felt in the positive correlations between the BIT and mean acceleration, and the negative correlations between the BIT and execution time. These data can be understood as showing that increased levels of VSN lead to copying at a slower and less varied speed consistent with uncertain movements' being coded by poor spatial information. They did not, however, show any evidence that specific effects resulted from damage to specific loci over and above those leading to VSN. In contrast, the opposite pattern of results with cube copying reflected the fact that patients with VSN drew fewer segments and therefore tended to give up sooner than SCs. These data support the view that cube copying is of particular difficulty for patients with $\mathrm{RH}$ brain damage, because they have specific difficulties in re-creating the third dimension (Griffiths, Cook, \& Newcombe, 1988). In fact, the present data suggest that these difficulties were restricted to patients with posterior RH brain damage, because no significant correlations were found for frontal/subcortical patients.

In summary, the static and dynamic data suggest that patients with VSN, especially those with posterior brain damage, create poor figure copies and do so using diagnostic movements. When one is copying representations of 2-D figures (squares, crosses), one's movements tend to be slow and relatively constant. When these 2-D shapes are stars, copying tends to move from left to right, in contrast to the pattern adopted by brain-damaged controls. In the copying of representations of 3-D cubes, fewer segments are drawn by patients with VSN than by SC patients and attempts at drawing tend to be short lived. What links both 2-D and 3-D cases is the suggestion that patients with VSN who produce recognizable representations of target figures do so while adopting a piecemeal approach to figure copying. Piecemeal copying strategies might result from some intrinsic aspect of VSN but might equally occur through related conditions caused by damage proximal to structures close to those whose damage leads to VSN. In other words, the relationship between piecemeal copying strategies and VSN is not simple and requires analysis that is beyond the scope and emphasis of the present paper.

We take the significant relationships between performance on the BIT and some of the measures obtained in this paper to indicate the utility of figure copying as a potential indicator of the severity of VSN. To this extent, aspects of figure copying might be thought of as duplicating information already made available through the BIT, although the testing time required by performance 
of the figure-copying tasks is much less than that required for performance of the BIT. By itself, time saving alone would represent a significant clinical advantage, but, in addition, our implementation of figure copying allows other advantages. First, the process through which figure copies are completed is made explicit-for example, where participants start copying relative to the figures that they are copying, and whether copying is performed "holistically" or in a piecemeal fashion. Second, although we have not pursued this in the present article, our implementation offers the opportunity to examine how the process of figure copying changes (or does not change) with recovery. The BIT alone does not make process information explicit at all and therefore cannot be used to examine process changes that occur with recovery.

Implementing the figure-copying task on a graphics tablet and employing algorithms within image analysis software allows the opportunity for more accurate specification of VSN (or VSN and its associated conditions). To this end, we have extended our work on performance in cancellation tasks. It is important to note, however, that the image analysis techniques described above are not restricted to analyzing patients with VSN. They could be used whenever populations might be supposed to differ in the use of spatial or constructional skills. In particular, they might be of use in the tracking of developmental change in children, who are thought to move from piecemeal to holistic perceptual strategies.

\section{REFERENCES}

Bradshaw, J. L., \& Mattingley, J. B. (1995). Clinical neuropsychology. San Diego: Academic Press.

Donnelly, N., Guest, R., Fairhurst, M., Potter, J., Deighton, A., \& PATEL, M. (1999). Developing algorithms to enhance the sensitivity of cancellation tests of visuospatial neglect. Behavior Research Methods, Instruments, \& Computers, 31, 668-673.

Goodale, M. A., Milner, A. D., Jakobson, L. S., \& Carey, D. P. (1990). Kinematic analysis of limb movements in neuropsychological research: Subtle deficits and recovery of function. Canadian Journal of Psychology, 44, 180-195.

Griffiths, K. M., CooK, M. L., \& Newcombe, R. L. G. (1988). Cube copying after cerebral damage. Journal of Clinical \& Experimental Neuropsychology, 10, 800-812.

KarNath, H.-O. (1994). Spatial limitations of eye movements during ocular exploration of simple line drawings in neglect syndrome. Cortex, 30, 319-330.

Marshall, R. S., Lazar, R. M., Binder, J. R., Desmond, D. W., DRUCKER, P. M., \& MOHR, J. P. (1994). Intrahemispheric localization of drawing dysfunction. Neuropsychologia, 32, 493-501.

Mattingley, J. B., Phillips, J. G., \& Bradshaw, J. L. (1994). Impairments of movement execution in unilateral neglect: A kinematic analysis of directional bradykinesia. Neuropsychologia, 32, 1111-1134.

Warrington, E. K., James, M., \& Kinsbourne, M. (1966). Drawing disability in relation to laterality of cerebral lesion. Brain, 89, 53-82. Williams, C. S. (1986). Designing digital filters. Englewood Cliffs, NJ: Prentice Hall.

Wilson, B. A., Cockburn, J., \& Halligan, P. W. (1987). Behavioural Inattention Test. Fareham, U.K.: Thames Valley Test Company.

(Manuscript received March 20, 2002;

revision accepted for publication October 26, 2003.) 